\title{
BMJ Open Mesh fixation technique for inguinal hernia repair: protocol for an umbrella review with integrated and updated network meta-analysis
}

\author{
Suphakarn Techapongsatorn (D) , ${ }^{1,2}$ Amarit Tansawet, ${ }^{1,2}$ Wisit Kasetsermwiriya, ${ }^{2}$ \\ Oraluck Pattanaprateep, ${ }^{1}$ Ammarin Thakkinstian ${ }^{1}$
}

To cite: Techapongsatorn S, Tansawet A, Kasetsermwiriya W, et al. Mesh fixation technique for inguinal hernia repair: protocol for an umbrella review with integrated and updated network meta-analysis. BMJ Open 2019;9:e031742. doi:10.1136/ bmjopen-2019-031742

- Prepublication history and additional material for this paper are available online. To view these files, please visit the journal online (http://dx.doi org/10.1136/bmjopen-2019031742).

Received 17 May 2019 Revised 03 September 2019 Accepted 30 September 2019

Check for updates

(C) Author(s) (or their employer(s)) 2019. Re-use permitted under CC BY-NC. No commercial re-use. See rights and permissions. Published by BMJ.

${ }^{1}$ Section for Clinical Epidemiology and Biostatistics, Faculty of Medicine Ramathibodi Hospital, Mahidol University, Bangkok, Thailand

${ }^{2}$ Department of Surgery, Faculty of Medicine Vajira Hospital, Navamindradhiraj University, Bangkok, Thailand

Correspondence to Prof Ammarin Thakkinstian; ammarin.tha@mahidol.ac.th

\section{ABSTRACT}

Introduction Inguinal hernia mesh repair is the standard care for symptomatic inguinal hernia. Mesh fixation is used to keep mesh in place for which various mesh fixation techniques have been used in open and laparoscopic inguinal hernia repair, but their effectiveness has remained inconclusive. Therefore, we developed a protocol for an umbrella review in order to summarise the evidences with integrate and update data of different mesh fixation techniques in both open and laparoscopic inguinal hernia repair.

Methods and analysis Previous systematic reviews and meta-analyses will be identified from Medline, Scopus, Cochrane Databases, EMBASE, Database of Abstracts of Reviews of Effects, PROSPERO Register, CINAHL, JBISRIS, EPPI-Centre, Wiley Online Library and Science Direct database. Two reviewers will independently determine studies for eligibility. Disagreement will be solved by consensus and arbitrated by the third reviewer. Data extraction will also be performed by two independent reviewers. For umbrella review, a descriptive analysis will be applied to describe evidence of mesh fixation effectiveness. Overlapping studies and excess significance test will be performed to assess whether previous evidences are bias. In addition, individual studies from meta-analysis and additional published studies will be pooled using network meta-analyses. We will use $\mathrm{I}^{2}$ statistic and Cochran's $Q$ test to assess heterogeneity. Risk of bias and publication bias, if appropriate, will be evaluated, as well as overall strength of the evidence. Ethics and dissemination This protocol has been registered with PROSPERO and approved with Institutional Review Board, Faculty of Medicine Vajira Hospital, Navamindradhiraj University, Bangkok, Thailand (COA 167/2018). The results will be published in peer-reviewed journal.

\section{PROSPERO registration number CRD42018111773}

\section{INTRODUCTION}

An inguinal hernia is the disease in which intraabdominal structures or parts of the intestine protrude though the triple triangle of the groin. This results in visible mass and pain, especially when the patients cough, stand for a period of time, bend over or lift
Strengths and limitations of this study

- This is the first report of an umbrella review in mesh fixation techniques for inguinal hernia repair.

- This study will summarise and integrate current evidences of clinical effectiveness produced by systematic reviews and meta-analyses.

- Quality and bias of current evidences will be assessed.

- All additional relevant published data will be updated and integrated with previous meta-analyses.

- Definitions and time assessment of hernia recurrence and chronic groin pain may be varied, working on summary data may be limited in exploring heterogeneity.

heavy objects. Although it is not seriously dangerous, it does not improve on its own, and might lead to life-threatening complications such as intestinal obstruction or strangulation. ${ }^{1}$

Inguinal hernia repair is regarded as the standard treatment for adult symptomatic inguinal hernia following the international guideline for groin hernia management in which mesh is used to reinforce inguinal floor. ${ }^{2}$ Surgical mesh repair can be performed by open or laparoscopic techniques. Lichtenstein repair is commonly applied for open approach whereas trans-abdominal preperitoneal repair (TAPP) and totally extraperitoneal repair (TEP) are commonly used for laparoscopic approach. ${ }^{2}$

Clinical effectiveness of hernia repair is usually concerned with hernia recurrence, chronic groin pain, length of hospitalisation, recovery time and complications, e.g., surgical site infection, seroma and hematoma. ${ }^{3-6}$ These outcomes are very dependent on surgical techniques, patient's factors and type of mesh/mesh fixation techniques. ${ }^{37}$ 
In mesh-based repair, flat mesh is recommended over three-dimension mesh, while self-gripping mesh is another alternative mesh. ${ }^{2}$ Several techniques for mesh fixations have been used including suture, glue or self-gripping mesh for open hernia repair (OHR); metallic tack, absorbable tack, glue, suture, self-gripping mesh or even non-fixation techniques for laparoscopic hernia repair (LHR). Up to date, there have been eight systematic reviews and meta-analyses (SRMAs) on $\mathrm{OHR}^{8-15}$ (ie, glue vs suture $(\mathrm{n}=5)^{89111214}$ and self-gripping mesh vs suture $(\mathrm{n}=3))^{101315}$ and one network meta-analysis (NMA). ${ }^{16}$ For LHR, comparisons were tack and glue $(\mathrm{n}=6),{ }^{17-22}$ fixation versus no fixation $(\mathrm{n}=4)^{23-25}$ and one $\mathrm{NMA}^{26}$.

Although evidences were interesting, the overall results were inconclusive, that is, five SRMAs suggested non-superiority in self-gripping mesh among all mesh fixations in OHR, ${ }^{10} 13151627$ whereas five SRMAs favoured glue fixation in decreasing postoperative pain. ${ }^{8} 9111214$ Among LHR, three SRMAs favoured glue fixation to decrease postoperative pain, ${ }^{171820}$ three SRMAs favoured no mesh fixation ${ }^{23-25}$ and one meta-study suggested all mesh fixations were comparable. ${ }^{19}$ In addition, clinical evidence in some aspects are still lacking, for example, whether clinical results of OHR and LHR are different given the same type of mesh fixations and also different mesh fixation techniques. Given availability of multiple SRMAs, we will therefore conduct an umbrella review to explore all available SRMAs and to summarise their findings for inguinal hernia repair. The updated data by combining individual studies from previous SRMAs with new published studies since the last SRMA will be pooled and analysed using NMA. These outcomes will provide recommendations for surgeons.

\section{METHODS AND ANALYSIS}

The protocol has been developed following the Preferred Reporting Items for Systematic Review and Meta-Analysis Protocols (PRISMA-P) guidelines ${ }^{28} 29$ with the PRISMA-P checklist provided in online supplementary file 1, and registered at PROSPERO. In addition, our umbrella review methods will follow suggestions proposed by Ioannidis ${ }^{30}$ and Aromataris et al. ${ }^{31}$ The review will be conducted during October 2018 to October 2019.

\section{Location of studies}

Relevant studies will be evaluated in an attempt to cover all available published studies, unpublished studies, ongoing studies, conference proceedings, theses and dissertations. The electronic databases will be used for searching include Scopus, Medline via PubMed, Cochrane Database of systematic reviews, EMBASE, Database of Abstracts of Reviews of Effects, PROSPERO Register, CINAHL, JBISRIS (JBI Database of systematic reviews and implementation reports), EPPI-Centre, Wiley Online Library and Science Direct published from January 2010 to June 2018. In addition, reference list of identified studies will also be checked for more relevant studies.

\section{Search strategies}

Search terms will be constructed as follows:

- Patients $(P)$ are adults who had uncomplicated inguinal hernia and underwent any inguinal hernia mesh repair (OHR, TEP and TAPP).

- Type of intervention $(I)$ and comparator $(C)$ could be any type of mesh fixation technique (ie, suture, glue and self-gripping mesh for open repair. Metallic tack, no fixation, absorbable tack, suture, glue and self-gripping mesh for laparoscopic repair).

- Outcome $(O)$ includes hernia recurrence, chronic groin pain, acute postoperative pain, operative time, recovery time (hospital stay, return to work or return to daily life), complication (seroma, hematoma, urinary retention).

- Study designs (S): systematic review and meta-analysis OR SRMAs.

These search terms will be combined within and between domains using conjunctions 'OR' and 'AND', respectively. The details of search terms are provided in online supplementary file 2.

In addition, individual studies will also be identified using the same search terms without specifying study design from the same databases indexes since the last search of the most recent SRMA.

\section{Selection of studies}

Studies published in English or other translatable languages using Google translate will be selected as follows.

\section{Umbrella review}

- SRMAs of randomised controlled trials (RCTs) of adult inguinal hernia who underwent OHR, TEP or TAPP repairs.

- Compared any pair of the mesh fixation techniques of OHR (ie, suture, glue and self-gripping mesh) or LHR (ie, tack, no fixation, glue, suture and self-gripping mesh).

- Pooled any efficacy outcome or adverse events of mesh fixations.

\section{Individual studies}

All individual studies that will be included in the selected SRMAs and also new published RCTs will be selected if they meet the following criteria:

- Studies in adults who underwent inguinal OHR, TEP or TAPP surgery.

- Comparison of any pair of mesh fixation techniques, that is, suture, glue and self-gripping mesh for OHR; tack, no fixation, glue, suture and self-gripping mesh for LHR.

- Having any of the following outcomes: hernia recurrence, chronic groin pain, operative time, hospital stay, return to work, return to daily life, seroma, hematoma, surgical site infection, urinary retention, postoperative pain.

- Have sufficient data for pooling. 


\section{Outcomes of interest}

The primary outcome of interests are hernia recurrence and chronic groin pain, which were defined according to original included studies.

The secondary outcomes are the postoperative pain, operative time, recovery time (hospital stay, return to work or return to daily life) and complication, for example, seroma, hematoma and urinary retention.

\section{Data extraction}

Data extraction forms/will be constructed and standardised before being applied. Two reviewers (ST and ATa) will independently extract data directly from the published studies. Any difference of extracted results will be discussed and consensus reached or adjudicated by supervisor (AT).

For umbrella reviews, the general characteristics data of SRMAs and their findings will be extracted separately by OHR, TEP and TAPP. For general characteristics data, the following data will be extracted: citation detail (study titles, principle author, journal, publication year), objective of the included study, type of SRMAs, number of included studies, period of searching, publication year of included studies, risk of bias assessment tools, characteristics of participants (eg, age, sex), type of interventions, type of interested outcome, number of included participants and method of synthesis. In addition, findings of SRMA will also be extracted including intervention-comparator, number of studies, number of participants, method of pooling and pooled effect size (ES) along with 95\% CI for each outcome, heterogeneity diagnostics (including $\tau^{2}$ and/or $\mathrm{I}^{2}$ and type of test along with p value), subgroup analysis if any, publication bias assessments, results (eg, Egger's test, funnel plot) and various sources of bias (conflict of interest, funding sources, reporting bias, overall quality of evidence and so on). Furthermore, individual included RCTs for each SRMA will be extracted (ie, first author, year, journal) to construct study-citation matrix across all SRMAs. Lastly, results of findings from each outcome and baseline risk/ incidence of each included RCT will also be extracted, which will be used to estimate power of test and then excess significance test.

For individual studies, data from the original individual studies will be extracted, which will be recorded in separate sheets for each outcome. This will include the data of authors, year of publication, intervention (mesh fixation), percent of male, mean age and SD, number of populations, number of events and number of no event.

\section{Quality assessment}

Methodological quality assessments will be performed separately by ST and ATa. Disagreements arising will be resolved by a third reviewer (AT). The quality assessment will be performed both for umbrella review and individual studies. Risk of bias in systematic reviews checklist $^{32}$ will be used for umbrella review concerning four domains: study eligibility criteria, methods used to identify and/or select studies, methods used to collect data and appraise studies, and regarding the synthesis/ findings. The Cochrane Collaboration's tool ${ }^{33}$ will be used for quality assessment in individual studies, which consists of six domains including sequence generation, allocation concealment, blinding, incomplete outcome data, selective outcome report and other sources of bias. Both quality assessment tools will be graded as low or high risk of bias if there is sufficient information to assess. Otherwise, it will be graded as unclear.

\section{Patient and public involvement}

No patient involvement

\section{Data analysis}

Data analysis will be performed as follows.

\section{Umbrella review}

Findings of SRMAs will be described separately by type of inguinal surgery (ie, open, TEP and TAPP) and outcomes. General characteristics, findings of intervention-comparator pair and outcomes will be described.

Bias of SRMAs evidences will be assessed as follows: first, a degree of overlapping across included SRMAs ${ }^{34}$ will be determined by creating study-citation matrix, in which each row will refer to the individual studies included in all SRMAs (index publications) and each column refers to individual SRMA (index reviews), see online supplementary file 3 . This citation matrix will be used to determine overlapping, that is, a number of the same study-citations across whole SRMAs. The degree of overlapping will be estimated using covered area (CA) and corrected covered area (CCA) using the following equations.

$$
\begin{aligned}
\mathrm{CA} & =\frac{\mathrm{N}}{\mathrm{r} * \mathrm{c}} \\
\mathrm{CCA} & =\frac{\mathrm{N}-\mathrm{r}}{\mathrm{rc}-\mathrm{r}}
\end{aligned}
$$

where $\mathrm{n}$ is the summation of the included publications (included overlapping) in all SRMAs, while $r$ and $c$ are row and column products of study-citation matrix. A CCA score is classified as slight, moderate and high overlap if the score $<5,5-15$ and $\geq 15$, respectively.

Second, an excess significance test ${ }^{35}$ will be assessed with the following equation.

$$
\chi^{2}=\frac{[\mathrm{O}-\mathrm{E}]^{2}}{\mathrm{E}}+\frac{[\mathrm{O}-\mathrm{E}]^{2}}{\mathrm{n}-\mathrm{E}}
$$

An excess significance test aims to evaluate a relative excess of formally significant findings in each individual study. For each SRMA and outcome, results (significance or non-significance at $\alpha$ level of 0.05 ) of individual included studies ( $\mathrm{n}$ ) will be extracted, and thus summation of these across total studies is the total number of observed positive results, that is, O. Power of test for each included study will be next estimated by assuming all included studies determining the same ES, that is, the pooled ES of that SRMA. Baseline risk according to that specific outcome will be extracted from each study, leading to estimate power of test given a total number of patients, a ratio of intervention per comparator and type 
1 error of 0.05 of each included study. Expected value of each included study will then be further estimated by multiplying power of test with the total number of patients. Summation of this across included studies will result in a total expected value $(\mathrm{E}) \cdot \chi^{2}$ statistic will be applied to test whether each SRMA has excess significance test result with a level of significance of $<0.10$. The details of study-citation matrix, formula to calculate percent of overlapping study, CA, CCA, and excess significant test will be provided in online supplementary file 3 .

\section{Integrated and undated data for network meta-analyses \\ Direct meta-analysis}

The efficacy of each pair of mesh fixations of OHR, TEP and TAPP will be directly compared and pooled for each outcome of interest if there are at least two studies for each comparison. Risk ratio (RR) will be estimated and pooled across studies using a fixed-effect model if heterogeneity is not present ( $\mathrm{p}$ value $<0.10$ or $\mathrm{I}^{2} \geq 25 \%$ ). Otherwise, a random-effect model will be applied. Heterogeneity will be assessed using Cochrane $Q$ test and $\mathrm{I}^{2}$ statistics. ${ }^{36-38}$ Source of heterogeneity will be explored by fitting covariables (ie, age, sex, BMI, hernia size and mean follow-up time,) one by one in a meta-regression model and subgroup analysis will be performed accordingly. Publication bias will be assessed using a funnel plot and Egger test. If one of these shows asymmetry, a contour enhanced funnel plot will be constructed to identify the cause of asymmetry.

\section{Network meta-analysis}

For each type of operation, NMA will be applied using a two-stage meta-analysis approach. In laparoscopic TEP or TAPP, interventions will be respectively coded as $1,2,3$, 4, 5 and 6 for metallic tack, no fixation, absorbable tack, suture fixation, glue and self-gripping mesh using metallic fixation as the reference group. In open repairs, interventions will be respectively coded as 1, 2 and 3 for suture, glue and self-gripping mesh using suture as the reference group. A network map will be constructed consisting of nodes and edges, and the nodes will be weighted by number of studies. In addition, a contribution plot will also be constructed to show the number of included studies pooled in direct and indirect comparisons.

Relative treatment effects (ie, coefficients or ln (RRs)) and variance-covariance will be estimated for each study. A multivariate meta-analysis with consistency model will be applied to pool $\ln (\mathrm{RRs})$ between studies. Multiple treatment contrasts will then be estimated among all possible fixation techniques.

The probability of being the best mesh fixation technique in lowering hernia recurrence and chronic groin pain will be estimated using surface under the cumulative ranking curve method; then the probability will be rank accordingly. Finally, inconsistency assumption (ie, difference between direct and indirect estimates) will be checked using a design-treatment interaction model. A predictive interval will be estimated considering uncertainty from heterogeneity and inconsistency of the network. All analyses will be performed using STATA software V.14.0; and $p$ values $<0.05$ will be considered statistically significant, except for heterogeneity where $\mathrm{p}<0.10$ will be used.

\section{DISCUSSION}

This study aims to use umbrella review methodology to synthesise available evidences from previous SRMAs. Quality of evidences, overlapping and excess significant test will be assessed. Umbrella review outcome will be summarising the evidence of mesh fixation techniques compared with SRMAs. In case that the evidence from umbrella reviews are controversial or have the new publication, we will repool data using individual studies that included in previous SRMAs plus additional recent published studies by applying NMA. The NMA is applied using a two-stage meta-analysis approach, in order to borrow information from common comparator to indirectly compare among interventions where studies with direct comparisons are still limited. In addition, potential optimal mesh fixations that yield highest efficacy and lowest complication will be assessed for within and also between each type of surgery. Summarise the evidence from umbrella review together with relative treatment effect together and treatment ranking from the NMA will be taken together for consideration as a conclusion for mesh fixation techniques suitable for inguinal hernia surgery. Our findings should be useful in development of clinical practice guidelines for hernia repairs and further economic evaluations.

\section{ETHICS AND DISSEMINATION}

Ethics approval for systematic review and meta-analysis is not required.

We will publish our findings in a peer-reviewed journal as well as disseminated through academic conference presentations. The data curation and deposition will be provided in supplementary documents.

In case of amendments to this protocol following its publication are needed, we will provide the date of each amendment, describe the change(s) and report the rationale for the change(s) in future publications arising from this protocol.

Acknowledgements This manuscript is a part of the Ph.D. (Clinical Epidemiology) training of Suphakarn Techapongsatorn, the Faculty of Medicine, Ramathibodi Hospital, Mahidol University and Faculty of Graduate Studies, Bangkok Thailand

Contributors ST is the principal investigator with overall responsibility for protocol development, together with AT. ST wrote the protocol and submitted the PROSPERO registration. ST, WK and ATa performed searching for relevant studies, data collection and data analysis. AT and OP provided help in designing, data analysis and editing of the manuscript. All authors read and approved the final manuscript.

Funding The authors have not declared a specific grant for this research from any funding agency in the public, commercial or not-for-profit sectors.

Competing interests ST is a PhD student of Clinical Epidemiology, Faculty of Medicine Ramathibodi Hospital and Faculty of Graduate Studies, Mahidol University. 
This study is a part of his dissertation which will be applied for granting graduation. The authors declare that they have no competing interests.

Patient consent for publication Not required.

Provenance and peer review Not commissioned; externally peer reviewed.

Open access This is an open access article distributed in accordance with the Creative Commons Attribution Non Commercial (CC BY-NC 4.0) license, which permits others to distribute, remix, adapt, build upon this work non-commercially, and license their derivative works on different terms, provided the original work is properly cited, appropriate credit is given, any changes made indicated, and the use is non-commercial. See: http://creativecommons.org/licenses/by-nc/4.0/.

ORCID iD

Suphakarn Techapongsatorn http://orcid.org/0000-0001-5373-7362

\section{REFERENCES}

1 Mehta A, Hutfless S, Blair AB, et al. Emergency department utilization and predictors of mortality for inpatient inguinal hernia repairs. J Surg Res 2017;212:270-7.

2 The HerniaSurge Group. International guidelines for groin hernia management. Hernia 2018;22:1-165.

3 Bhangu A, Singh P, Pinkney T, et al. A detailed analysis of outcome reporting from randomised controlled trials and meta-analyses of inguinal hernia repair. Hernia 2015;19:65-75.

4 Cavazzola LT, Rosen MJ. Laparoscopic versus open inguinal hernia repair. Surg Clin North Am 2013;93:1269-79.

5 Vărcuș F, Duță C, Dobrescu A, et al. Laparoscopic repair of inguinal hernia TEP versus TAPP. Chirurgia 2016;111:308-12.

6 Campanelli G, Bruni PG, Morlacchi A, et al. Primary inguinal hernia: the open repair today pros and cons. Asian J Endosc Surg 2017:10:236-43.

7 Aquina CT, Fleming FJ, Becerra AZ, et al. Explaining variation in ventral and inguinal hernia repair outcomes: a population-based analysis. Surgery 2017;162:628-39.

8 Colvin HS, Rao A, Cavali M, et al. Glue versus suture fixation of mesh during open repair of inguinal hernias: a systematic review and metaanalysis. World J Surg 2013;37:2282-92.

9 de Goede B, Klitsie PJ, van Kempen BJH, et al. Meta-analysis of glue versus sutured mesh fixation for Lichtenstein inguinal hernia repair. Br J Surg 2013;100:735-42.

10 Ismail A, Abushouk Al, Elmaraezy A, et al. Self-Gripping versus sutured mesh fixation methods for open inguinal hernia repair: a systematic review of clinical trials and observational studies. Surgery 2017;162:18-36.

11 Lin H, Zhuang Z, Ma T, et al. A meta-analysis of randomized control trials assessing mesh fixation with glue versus suture in Lichtenstein inguinal hernia repair. Medicine 2018;97:e0227.

12 Liu H, Zheng X, Gu Y, et al. A meta-analysis examining the use of fibrin glue mesh fixation versus suture mesh fixation in open inguinal hernia repair. Dig Surg 2014;31:444-51.

13 Sajid MS, Farag S, Singh KK, et al. Systematic review and metaanalysis of published randomized controlled trials comparing the role of self-gripping mesh against suture mesh fixation in patients undergoing open inguinal hernia repair. Updates Surg 2014;66:189-96.

14 Sun $\mathrm{P}$, Cheng X, Deng S, et al. Mesh fixation with glue versus suture for chronic pain and recurrence in Lichtenstein inguinal hernioplasty. Cochrane Database Syst Rev 2017;2.

15 Zhang C, Li F, Zhang H, et al. Self-gripping versus sutured mesh for inguinal hernia repair: a systematic review and meta-analysis of current literature. J Surg Res 2013;185:653-60.

16 Rausa E, Asti E, Kelly ME, et al. Open inguinal hernia repair: a network meta-analysis comparing self-gripping mesh, suture fixation, and glue fixation. World J Surg 2019;43:447-56.

17 Antoniou SA, Köhler G, Antoniou GA, et al. Meta-Analysis of randomized trials comparing nonpenetrating vs mechanical mesh fixation in laparoscopic inguinal hernia repair. Am J Surg 2016;211:239-49.

18 Li J, Ji Z, Zhang W. Staple fixation against adhesive fixation in laparoscopic inguinal hernia repair: a meta-analysis of randomized controlled trials. Surg Laparosc Endosc Percutan Tech 2015;25:471-7.

19 Sajid MS, Ladwa N, Kalra L, et al. A meta-analysis examining the use of tacker mesh fixation versus glue mesh fixation in laparoscopic inguinal hernia repair. Am J Surg 2013;206:103-11.

20 Shah NS, Fullwood C, Siriwardena AK, et al. Mesh fixation at laparoscopic inguinal hernia repair: a meta-analysis comparing tissue glue and tack fixation. World J Surg 2014;38:2558-70.

21 Kaul A, Hutfless S, Le H, et al. Staple versus fibrin glue fixation in laparoscopic total extraperitoneal repair of inguinal hernia: a systematic review and meta-analysis. Surg Endosc 2012;26:1269-78.

22 Shi Z, Fan X, Zhai S, et al. Fibrin glue versus staple for mesh fixation in laparoscopic transabdominal preperitoneal repair of inguinal hernia: a meta-analysis and systematic review. Surg Endosc 2017;31:527-37.

23 Sajid MS, Ladwa N, Kalra L, et al. A meta-analysis examining the use of tacker fixation versus no-fixation of mesh in laparoscopic inguinal hernia repair. Int $J$ of Surgery 2012;10:224-31.

24 Tam K-W, Liang H-H, Chai C-Y. Outcomes of staple fixation of mesh versus nonfixation in laparoscopic total extraperitoneal inguinal repair: a meta-analysis of randomized controlled trials. World J Surg 2010;34:3065-74.

25 Teng YJ, Pan SM, Liu YL, et al. A meta-analysis of randomized controlled trials of fixation versus nonfixation of mesh in laparoscopic total extraperitoneal inguinal hernia repair. Surg Endosc 2011;25:2849-58.

26 Techapongsatorn S, Tansawet A, Kasetsermwiriya W, et al. Mesh fixation technique in totally extraperitoneal inguinal hernia repair - A network meta-analysis. Surgeon 2019;17.

27 Ladwa N, Sajid MS, Sains P, et al. Suture mesh fixation versus glue mesh fixation in open inguinal hernia repair: a systematic review and meta-analysis. Int J Surg 2013;11:128-35.

28 Moher D, Shamseer L, Clarke M, et al. Preferred reporting items for systematic review and meta-analysis protocols (PRISMA-P) 2015 statement. Syst Rev 2015;4:1.

29 Shamseer L, Moher D, Clarke M, et al. Preferred reporting items for systematic review and meta-analysis protocols (PRISMA-P) 2015: elaboration and explanation. BMJ 2015;349:97647.

30 loannidis JPA. Integration of evidence from multiple meta-analyses: a primer on umbrella reviews, treatment networks and multiple treatments meta-analyses. Can Med Assoc J 2009;181:488-93.

31 Aromataris E, Fernandez R, Godfrey CM, et al. Summarizing systematic reviews: methodological development, conduct and reporting of an umbrella review approach. Int J Evid Based Healthc 2015;13:132-40.

32 Whiting P, Savović J, Higgins JPT, et al. ROBIS: a new tool to assess risk of bias in systematic reviews was developed. J Clin Epidemiol 2016;69:225-34.

33 Armijo-Olivo S, Stiles CR, Hagen NA, et al. Assessment of study quality for systematic reviews: a comparison of the Cochrane collaboration risk of bias tool and the effective public health practice project quality assessment tool: methodological research. J Eval Clin Pract 2012;18:12-18.

34 Pieper D, Antoine S-L, Mathes T, et al. Systematic review finds overlapping reviews were not mentioned in every other overview. $J$ Clin Epidemiol 2014;67:368-75.

35 loannidis JPA, Trikalinos TA. An exploratory test for an excess of significant findings. Clin Trials 2007;4:245-53.

36 Dawson DV, Pihlstrom BL, Blanchette DR. Understanding and evaluating meta-analysis. J Am Dent Assoc 2016;147:264-70.

37 Garcia-Alamino JM, Bankhead C, Heneghan C, et al. Impact of heterogeneity and effect size on the estimation of the optimal information size: analysis of recently published meta-analyses. BMJ Open 2017;7:e015888.

38 Baker WL, Michael White C, Cappelleri JC, et al. Understanding heterogeneity in meta-analysis: the role of meta-regression. Int $\mathrm{J}$ Clin Pract 2009;63:1426-34. 